\title{
Atividades de promoção de saúde desenvolvidas por acadêmicos de Odontologia: relato de experiência
}

\section{Health promotion activities developed by undergraduate dental students: report of an experience Actividades de promoción de la salud desarrolladas por estudiantes universitarios de Odontología: reporte de una experiencia} José Henrique de Araújo $\mathbf{C R U Z}^{\mathbf{1}}$

Elaine Roberta Leite de SOUZA ${ }^{1}$

Nílvia Maria Lima GOMES ${ }^{1}$

Laise da Luz RAMOS ${ }^{1}$

Itamar da Silva NUNES ${ }^{1}$

André Paulo Gomes SIMÕES

Eric Marcelino GUEDES ${ }^{1}$

Basílio Rodrigues VIEIRA ${ }^{2}$

Moan Jéfter Fernandes COSTA ${ }^{3}$

Faldryene de Sousa QUEIROZ ${ }^{4}$

${ }^{1}$ Acadêmico(a), Curso de Graduação em Odontologia. Centro de Saúde e Tecnologia Rural, Universidade Federal de Campina Grande, UFCG 58708-110 Patos-PB, Brasil

${ }^{2}$ Mestrando do Programa de Pós-Graduação em Odontologia pela Universidade Federal da Paraíba - UFPB, João Pessoa-PB, Brasil

${ }^{3}$ Doutorando no Programa de Pós-Graduação em Odontologia pela Universidade Federal do Rio Grande do Norte - UFRN, Natal-RN, Brasil

${ }^{4}$ Professor(a) Doutor(a), Curso de Graduação em Odontologia. Centro de Saúde e Tecnologia Rural, Universidade Federal de Campina Grande, UFCG 58708-110 Patos-PB, Brasil

\section{Resumo}

Introdução: Sabendo que a saúde bucal está intimamente ligada à saúde geral e à qualidade de vida, ações em Educação em Saúde Bucal são de extrema importância no incentivo à prática de higiene oral da população. Objetivo: Relatar duas experiências da interação serviço-ensino vivenciado por estudantes de Odontologia, demonstrando os artifícios utilizados para realização de atividades práticas do componente curricular Estágio Supervisionado em Sistema Público de Saúde I, do curso de Odontologia da UFCG. Metodologia: Foram realizadas visitas de reconhecimento do local e planejamento das atividades que poderiam ser executadas, incluindo público-alvo, melhor horário para realização e planejamento multiprofissional para desenvolvimento das ações. A estratégia metodológica utilizadas para as atividades de educação em saúde bucal, enfatizam-se para a Academia da Saúde orientações em saúde bucal, prevenção do câncer de boca e fatores predisponentes, orientação nutricional e atividade física de dança, destacando a influência que ela possui com a saúde oral. E nas abordagens à Creche, destacam-se as brincadeiras lúdicas de teatro, atividade de desenhos para colorir, musicoterapia e escovação em macro-modelo odontológico. Relato de experiência: Os acadêmicos de Odontologia puderam perceber seu valor para a transformação de sorrisos, contribuíram para modificar o quadro de saúde de intervencionista preventivo e tornaram a atitude voluntária de ações sociais em atividades contínuas nessa e em outras creches da cidade. Conclusão: Para os alunos dos cursos de saúde, é imprescindível uma formação mais ampla, em ambientes diferenciados, com experiências inovadoras, atividades coletivas e não somente a prática tradicional realizada em clínica de ensino.

Descritores: Apoio ao Desenvolvimento de Recursos Humanos; Saúde Bucal; Odontologia; Atenção Primária à Saúde.

\section{Abstract}

Introduction: Knowing that oral health is closely linked to general health and quality of life, actions in Oral Health Education are extremely important in encouraging the oral hygiene practice of the population. Objective: To report two experiences of the service-teaching interaction experienced by students of Dentistry, demonstrating the artifice used to perform practical activities of the curricular component Supervised Stage in Public Health System I of the UFCG Dentistry course. Methodology: Site reconnaissance visits and planning of the activities that could be carried out, including target audiences, better time for realization and multiprofessional planning for actions development were carried out. The methodological strategy used for oral health education activities emphasizes for the Health Academy guidelines on oral health, mouth cancer prevention and predisposing factors, nutritional orientation and physical activity of dance, highlighting the influence it has with oral health. And in the approaches to the nursery, we can highlight the playful play of the theater, activity of coloring drawings, music therapy and brushing in a macro-dental model. Experience report: Dentistry students were able to perceive their value for the transformation of smiles, contributed to modify the preventive interventionist health framework, and turned the voluntary attitude of social actions into continuous activities in this and other day care centers in the city. Conclusion: For the students of the health courses, a broader education is essential, in differentiated environments, with innovative experiences, collective activities and not only the traditional practice in a teaching clinic.

Descriptors: Training Support; Oral Health; Dentistry; Primary Health Care.

\section{Resumen}

Introducción: Sabiendo que la salud bucal está íntimamente ligada a la salud general ya la calidad de vida, acciones en Educación en Salud Bucal son de extrema importancia en el incentivo a la práctica de higiene oral de la población. Objetivo: Informar dos experiencias de la interacción servicioenseñanza vivido por estudiantes de Odontología, demostrando los artificios utilizados para realización de actividades prácticas del componente curricular Etapa Supervisada en Sistema Público de Salud I, del curso de Odontología de la UFCG. Metodología: Se realizaron visitas de reconocimiento del local y planificación de las actividades que podrían ser ejecutadas, incluyendo público objetivo, mejor horario para realización y planificación multiprofesional para el desarrollo de las acciones. La estrategia metodológica utilizada para las actividades de educación en salud bucal, se enfatiza para la Academia de la Salud orientaciones en salud bucal, prevención del cáncer de boca y factores predisponentes, orientación nutricional y actividad física de danza, destacando la influencia que posee con la salud oral. Y en los abordajes a la Guardería, se destacan los juegos lúdicos de teatro, actividad de dibujos para colorear, musicoterapia y cepillado en macro modelo odontológico. Relato de experiencia: Los académicos de Odontología pudieron percibir su valor para la transformación de sonrisas, contribuyeron a modificar el cuadro de salud de intervencionista preventivo y tornaron la actitud voluntaria de acciones sociales en actividades continuas en esa y en otras guarderías de la ciudad. Conclusión: Para los alumnos de los cursos de salud, es imprescindible una formación más amplia, en ambientes diferenciados, con experiencias innovadoras, actividades colectivas y no sólo la práctica tradicional realizada en clínica de enseñanza.

Descriptores: Prótesis Dental; Estética Dental; Cerámica; Coronas con Frente Estético.

INTRODUÇÃO

A proposta do olhar integral ao paciente requer profissionais da saúde habilidosos na atenção humanizada e interpretação da conjuntura do processo saúde-doença do indivíduo ${ }^{1,2}$.As mudanças no perfil epidemiológico das doenças bucais, as novas práticas baseadas em evidencias cientificas, e principalmente a promoção de saúde no seu conceito ampliado, exigem a formação de um profissional generalista, tecnicamente competente e com sensibilidade social com foco nas famílias para trabalhar no Setor público que apresenta a Unidade Básica de Saúde (UBS) como reorientadora destes 
serviços $^{3,4}$.

A assistência generalista e humanizada necessita do ensino que promova experiências no serviço de saúde, com integração estudanteprofissional, por meio de troca de saberes problematizadores, na busca de um modelo de atenção preventivo e promotor da saúde, com contribuições da gestão e controle social ${ }^{5,6}$.

As atividades extramuros são essencialmente importantes para a esfera acadêmica e também para as comunidades participantes, já que engloba o público alvo tornando-os participantes do processo de produção de saúde desenvolvido pela universidade ${ }^{7}$. $\mathrm{O}$ desafio primordial consiste em gerar e implantar estratégias que possibilitem integrar em seu entorno a comunidade, transformando-as em participantes de projetos de mudança, inclusão social e desenvolvimento. É de grande importância destacar a função da Universidade diante da sociedade, tornando possível uma reflexão crítica para sua transformação, bem como possibilitar a materialização do princípio constitucional de inseparabilidade entre ensino, pesquisa e extensão ${ }^{8}$.

O serviço público na Atenção Básica frequentemente representa um primeiro passo para a vida profissional, sendo pouco valorizado pelo recém-formado, que tem forte tendência à especialização ${ }^{9}$. O estudante põe em prática o conhecimento adquirido durante a graduação em conformidade com a realidade, desenvolvendo ética e profissionalismo. Nesse raciocínio, essas atividades podem contribuir para formação de cirurgiõesdentistas mais capacitados, humanizados, com facilidade para trabalhar em equipes multiprofissionais e entender às necessidades da comunidade as quais estarão inseridos ${ }^{10}$.

Desta maneira, este fato caminha junto com os novos objetivos de formação estabelecidos nas Diretrizes Curriculares Nacionais (DCN) para os cursos de graduação em Odontologia, buscando a formação de um profissional generalista, com sólida formação técnico-científica, humanística e ética, guiado para a promoção de saúde ${ }^{11}$.

Diante do exposto, o objetivo deste estudo foi compartilhar as experiências institucionais desenvolvidas pelos graduandos do quinto período do curso de Odontologia da Universidade Federal de Campina Grande (UFCG), campus Patos/PB, na Academia da Saúde vinculada Núcleo de Apoio a Saúde da Família (NASF) e em uma Creche do município de Patos-PB, Brasil.

\section{MATERIAL E MÉTODO}

A Disciplina de Estágio Supervisionado em Sistema Público de Saúde I do curso de Odontologia da Universidade Federal de Campina Grande (UFCG) Campus Patos-PB, tem como objetivos: proporcionar ao aluno contato com as atividades de atenção à saúde na comunidade, reconhecendo a realidade local; realizar busca ativa de espaços de atores sociais, baseados nos conceitos de clínicaampliada bem como, promover uma reflexão do processo de trabalho em uma comunidade, destacando a contribuição do trabalho em equipe e a intervenção direta na promoção da Saúde Coletiva, com vistas à organização e a otimização dos resultados da assistência a saúde.

As atividades foram elaboradas $\mathrm{e}$ desenvolvidas voluntariamente ao longo dos meses de fevereiro e março de 2017, por sete alunos do quinto período na disciplina de Estágio Supervisionado em Sistema Público de Saúde I do curso de Odontologia da UFCG, Campus Patos/PB, sob a orientação de professores e em colaboração com técnicos administrativos em educação e serviços de saúde do setor público e privado. O presente trabalho abordará a experiência em dois locais escolhidos para realização das atividades propostas pela disciplina: A Academia da Saúde vinculada Núcleo de Apoio a Saúde da Família (NASF) e em uma Creche do município de Patos-PB, Brasil.

Inicialmente, foram realizadas visitas de reconhecimento do local e planejamento das atividades que poderiam ser executadas, incluindo público alvo, melhor horário para realização das atividades e planejamento multiprofissional para melhor desenvolvimento das atividades. $\mathrm{Na}$ Academia de Saúde localizada no Bairro Jatobá da cidade de Patos, o público alvo consistiu de 25 adultos jovens e 30 idosos cadastrados no NASF, todos do sexo feminino, além da Educadora Física da Academia. As atividades foram desenvolvidas em duas vertentes: uma voltada para as adultas jovens e outra para as idosas, em único encontro em cada vertente. Na Creche, selecionada para aplicação o de ações de promoção de saúde bucal, também localizada no Bairro Jatobá, cujo público alvo era composto em média por 40 crianças do primeiro ano da pré-escola e faixa etária média de seis anos de idade, de ambos os sexos, a abordagem foi realizada em um único encontro.

Como elementos da estratégia metodológica utilizadas para as atividades de educação em saúde bucal, enfatizam-se para a Academia da Saúde orientações em saúde bucal, prevenção do câncer de boca e fatores predisponentes, orientação nutricional e atividade física de dança, destacando a influência que ela possui com a saúde bucal. E nas abordagens à Creche, destacam-se as brincadeiras lúdicas de teatro, atividade de desenhos para colorir, musicoterapia e escovação em macromodelo odontológico.

\section{RELATO DE EXPERIÊNCIA}

- Planejamento em Saúde

$\mathrm{O}$ início da atividade extramuro proposta

pelos acadêmicos começou por uma visita à 
Academia de Saúde e à Creche objetivando reconhecer o local de atuação e a rotina de trabalho da Educadora Física da Academia da Saúde e Professores da Creche, permitindo a criação do primeiro vínculo com os profissionais.

O planejamento das atividades que pudessem ser executadas, incluindo público alvo, melhor horário para realização das atividades e planejamento multiprofissional para enriquecer os olhares do que estava sendo proposto, teve um papel fundamental para o sucesso das atividades.

\section{- Experiência na Academia da Saúde}

Num segundo momento, destinado à elaboração e aplicação de ações sociais na Academia da Saúde direcionadas às mulheres adultas jovens, houve um encontro interprofissional entre os graduandos do curso de Odontologia, a Educadora Física da academia e o público alvo, buscando frisar a relação entre o bem estar bucal e o bem estar sistêmico, uma vez que a saúde bucal é uma porção integrante da saúde geral e deveria ser visto como componente essencial para os programas de saúde sistêmica, devendo sempre fornecer alguns meios de satisfazer as necessidades de saúde bucal (Figura 1).

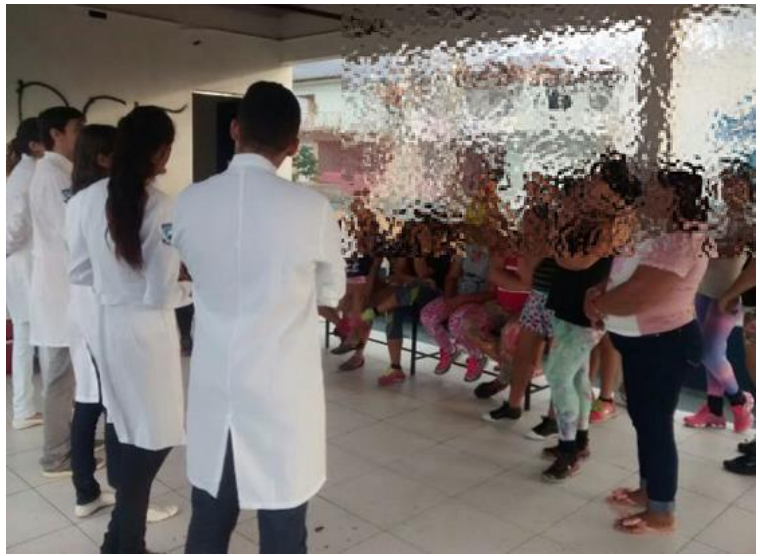

Figura 1: Importância da saúde bucal para a saúde sistêmica.

Nesse sentido, foram feitas orientações sobre câncer bucal associado a fatores predisponente tais como o tabagismo, consumo de bebidas alcoólicas e exposição aos raios solares (raios ultravioletas) sem a devida proteção. Destacou-se a importância de avaliar a própria boca e frisar as visitas periódicas ao cirurgião-dentista, uma vez que ocorre o negligenciamento de quaisquer alterações em lábio, palato, bochechas, gengivas, língua e soalho bucal, evidenciando a total atenção da sociedade para com os dentes, um erro comumente cometido em regiões mais carentes (Figura 2). Além disso, enfatizaram-se orientações sobre uso de prótese dentária para todas aquelas possuíam uma, seja ela total ou parcial removível, quanto ao tempo de uso, higienização e precauções que devem ser tomadas.

Ainda, foi preparada uma coreografia de Zumba em conjunto com a Educadora Física para não fugir do real objetivo (Figura 3) e um lanche após a atividade física, contendo salada de frutas, suco de abacaxi com hortelã, melão, melancia, bananas, visto que o consumo de alimentos saudáveis e naturais é melhor que alimentos ricos em açúcar e pegajosos aos dentes (Figura 4).

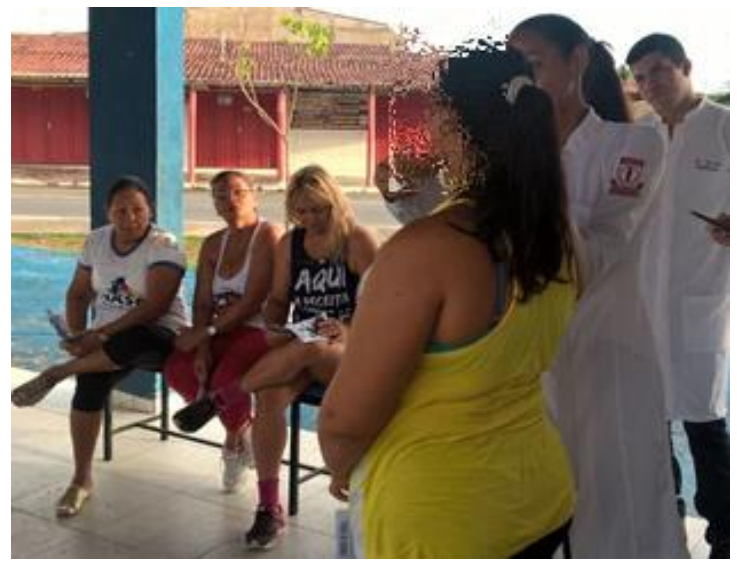

Figura 2: Importância de conhecer a própria boca

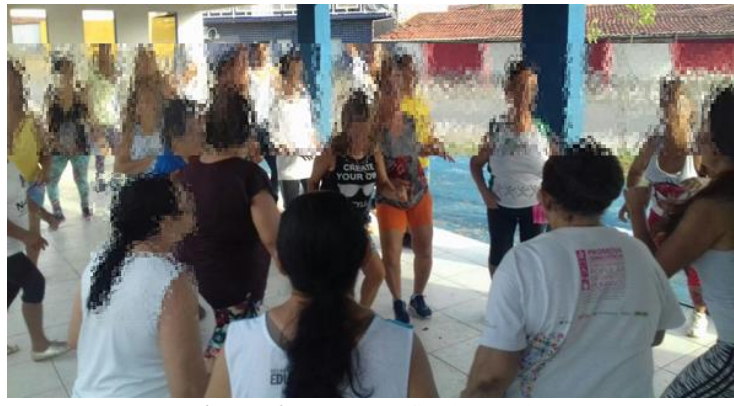

Figura 3: Coreografia de zumba.

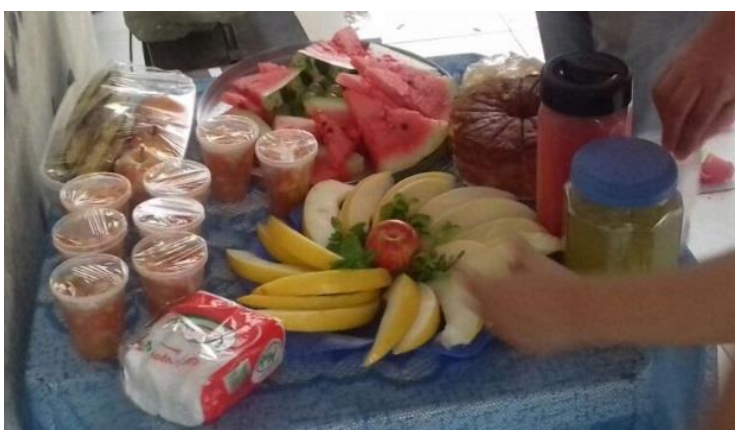

Figura 4: Orientação nutricional.

Para o público de idosas, é necessário conhecer as alterações fisiológicas e patológicas que acometem o organismo do sujeito idoso e o papel da Odontologia em relação a essa faixa populacional e de mantê-las em condições de saúde bucal que não comprometam a alimentação normal nem tenham repercussões negativas sobre a saúde geral e sobre o estado psicológico do indivíduo. Além das informações e dinâmicas supracitadas para o grupo de adultas jovens serem também aplicadas paras as idosas, enfatizou-se a repercussão que o Diabetes tipo I e II e a Hipertensão têm sobre a cavidade bucal e sistêmica.

Diante disso, em ambos os grupos de jovens adultas e idosas houve efetivo resultado, elas se mostraram curiosas com os assuntos que foram expostos, tiraram dúvidas que traziam consigo, além de terem sido bem receptivas, tendo em vista a carência de ações sociais e divulgação de informação 
a respeito de saúde bucal. Os alunos participantes das ações na Academia da Saúde tiveram suas expectativas atingidas e concluíram que a participação do graduando e do cirurgião-dentista é de fundamental importância para transformar a realidade das comunidades carentes, depositando nelas o que foi aprendido em sala de aula e que a Universidade tem responsabilidade no ambiente em que está inserida, uma vez que através da educação, pesquisa e extensão é possível assinalar e intervir nas mazelas sociais.

\section{- Experiência na Creche}

Posteriormente às ações da Academia da Saúde, destinou-se às atividades na creche para aplicação de trabalhos educativas com ênfase em saúde bucal, abordando-se a primeira turma da préescola totalizando em média 40 crianças com idade média de 6 anos. Para isso, com objetivo de atingir a pouca idade das crianças, preferiu-se ações educativas que prendessem a atenção delas, com dinâmicas sempre voltadas à escovação e higiene bucal.

Iniciou-se a ação social com dois dos graduandos vestidos de elemento dentário confeccionados com Folhas de EVA para artesanato, permitindo a interação delas com os acadêmicos de Odontologia e facilitando o retorno positivo por elas (Figura 5). Posteriormente, uma atividade de perguntas e respostas a respeito dos alimentos saudáveis e não saudáveis à dentição, fazendo uso de figuras em desenho para que elas mesmas pudessem colar na roupa de dente dos graduandos confeccionada em EVA aquelas imagens que elas julgassem saudáveis (Figura 6).

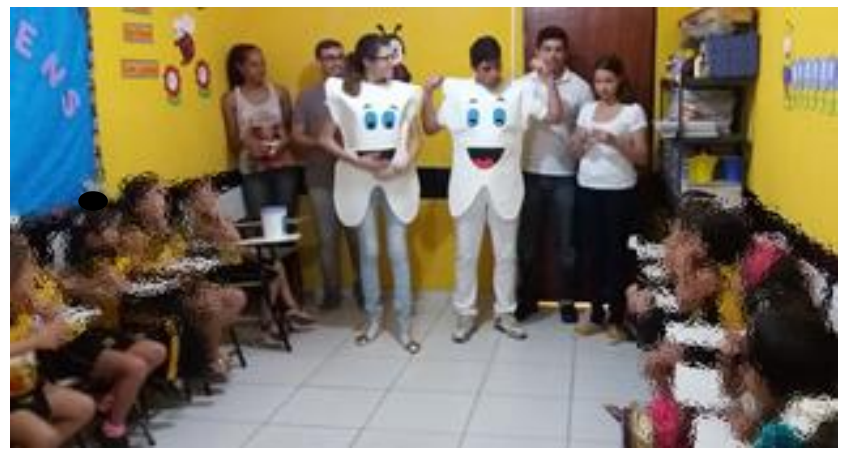

Figura 5: Acadêmicos vestidos com roupas de dentes.

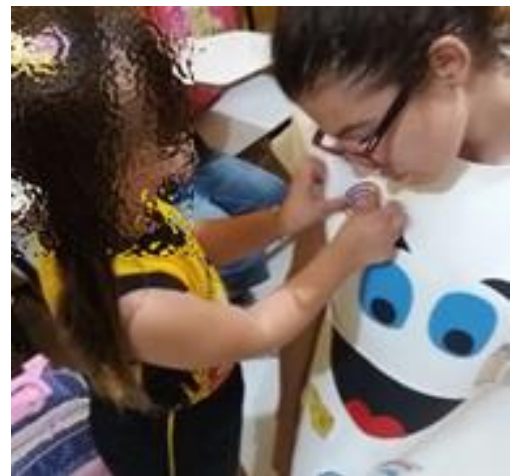

Figura 6: Colagem de figuras de alimentos saudáveis e não saudáveis.
$\mathrm{Na}$ sequência, efetuou-se a atividade de pintura de rostos com tinta facial (Color Make, São Paulo/SP) para não gerar irritação à pele das crianças e elas pudessem desfrutar do momento de descontração (Figura 7); e pintura de desenhos educativos com foco na escovação dentária, sempre explicando para cada uma delas a importância da escovação tão precocemente, com frequência e horário de escovação de três vezes ao dia, todos os dias, uma vez pela manhã, tarde e noite, tornando o momento de orientação à higiene bucal em um espaço de diversão.

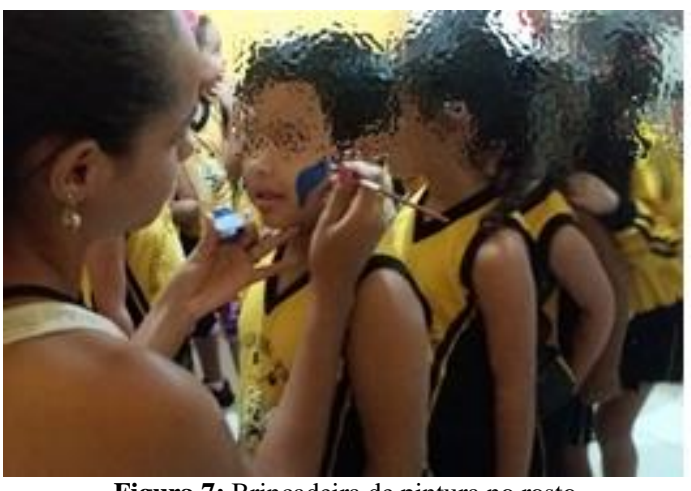

Figura 7: Brincadeira de pintura no rosto.

Ainda foi possível supervisionar a escovação, associada à musicoterapia, praticada pelas próprias crianças no macromodelo odontológico, utilizando escova dentária para demonstração da forma correta de escovação e das partes da cavidade bucal que devem ser higienizadas, possibilitando educa-las quanto à importância da higiene (Figura 8).

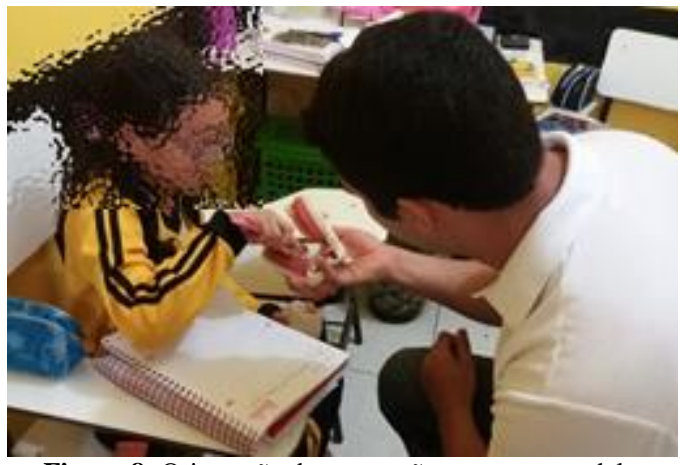

Figura 8: Orientação de escovação em macromodelo.

A partir das práticas sociais nessa creche, portanto, pode-se observar a carência das crianças por atenção social, educativa e de higiene bucal, uma vez que poucas visitas deste gênero foram feitas ao longo do ano letivo delas. Além disso, embora os préescolares já tivessem tido alguma instrução sobre a escovação adequada e diária três vezes ao dia, notouse falta de incentivo e motivação contínua pelos pais, já que elas afirmavam escovar os dentes apenas uma vez ao dia.

Grande parte das crianças que participaram se mostraram receptivas, cooperativas e assíduas diante das atividades propostas pelo grupo de graduandos, demonstrando interesse por uma segunda oportunidade em recebê-los. Os acadêmicos 
em Odontologia puderam perceber seu valor para a transformação de sorrisos, modificar o quadro de saúde intervencionista para preventivo e tornar a atitude voluntária de ações sociais em atividades contínuas nessa e em outras creches da cidade.

\section{DISCUSSÃO}

Promover e orientar em saúde significa colaborar para mudanças no estilo de vida, auxiliando o conhecimento e, como resultado, propiciando o bem-estar físico e emocional ${ }^{12}$. Como posto por Ricci et al. ${ }^{13}$, dificuldades em manter a atenção das pessoas e despertar o seu interesse já eram esperados, desse modo, buscou-se inserir as informações indispensáveis através de conversas curtas e simplificadas para uma melhor compreensão, estimando a utilização de ilustrações didáticas, o que torna mais dinâmico e produtivo, além disso, as atividades com os idosos tem se mostrado efetivas quando realizada em grupos menores, visto que grupos maiores poderiam se dispersar e tornar a atividade improdutiva.

Os conhecimentos adquiridos na universidade pelos acadêmicos de odontologia e reproduzidos para o grupo de idosas na academia de saúde corroboram com os conceitos de Ricciet al. ${ }^{13}$, uma vez que esperando a pouca atenção delas, podese planejar, previamente, ações que tivessem um caráter dinâmico, curta duração em palestras e fácil entendimento.

Para Pereira et al. ${ }^{14}$ as práticas desenvolvidas externamente ao meio universitário favorecem interações mais dinâmicas e verdadeiras entre os estudantes e as comunidades, beneficiando a ambos.

A aplicação de conceitos aprendidos e debatidos, por meio das atividades de promoção de saúde, permitiu tratar de forma ampliada a realidade desses grupos, podendo contribuir para melhoria da qualidade de vida dos indivíduos envolvidos. Atividades como essas são capazes de sensibilizar os alunos frente à realidade social na qual atuam e contribuem para a sua formação profissional ${ }^{15}$.

Segundo Domingues et al. ${ }^{16}$, realizar atividades que vão além dos conhecimentos teóricos específicos, permitindo atingir experiências importantes para o desenvolvimento pessoal e profissional. Estas atividades mostram diferentes potencialidades para o amadurecimento dos alunos, auxiliam no desenvolvimento da comunicação, favorecem o estabelecimento de contatos, estabelecem um diálogo entre a teoria e a prática e induzem a tomada de decisões.

Foi constatado a partir da ação feita em sala de aula o interesse e a participação dos alunos nas atividades desenvolvidas. Isso mostra que a sala de aula é, adequadamente, um espaço social para se trabalhar conteúdos associados à educação em Saúde e a Higiene Bucal junto dos pré-escolares, evidenciando as potencialidades do acadêmico de graduação no ambiente fora da Universidade.

A constatação da vulnerabilidade de determinados grupos populacionais requer das instituições sociais, representadas pela universidade, escola e serviços de saúde, um esforço integrado de várias esferas para contemplar o preceito constitucional da universalidade de acesso à saúde, resgatando os princípios da integralidade e da equidade como possibilidades de diminuir as injustiças sociais ${ }^{7}$.

De acordo com Loretto et al. $^{17}$, uma instituição pesquisada apresentou seu modelo de formação no qual se incluia Clínica de Atenção Básica como primeira experiência clínica do aprendiz, situada no $5^{\circ}$ período do curso, e concluiu que houve a melhoria na triagem. A interdisciplinaridade, o compromisso e competência docente são as melhorias mais exigidas pelos alunos; e o fortalecimento da interdisciplinaridade e a organização dosprontuários são as melhorias mais exigidas pelos docentes. Nesse sentido, avançamos quanto a uma nova proposta de atendimento que uniu a teoria com as práticas, técnicas tão importantes para formação profissional dos acadêmicos.

Com o relato de experiência, é possível mudar o cenário de atenção em saúde, esperar um profissional egresso com uma nova postura, com compromissos éticos com a sociedade, ajudando o usuário a viver com saúde e que possa atuar em equipes multidisciplinares ${ }^{18}$. Desta forma, os aspectos expostos confirmam com o que foi vivenciado pelos acadêmicos do curso de Odontologia da UFCG mediante as ações de promoção de saúde na Academia da Saúde e na Creche, que ações sociais deste caráter têm a contribuir para a formação profissional.

\section{CONSIDERAÇÕES FINAIS}

As atividades desenvolvidas dentro do componente curricular basearam-se na atenção básica, através de práticas que visam garantir os princípios da integralidade, universalidade e equidade. As tarefas propiciaram um maior conhecimento por parte dos alunos que transformaram os conhecimentos acadêmicos em ações, almejando sempre atingir diversos públicos, através de trabalhos com ciclos de vida, valorizando cada etapa do desenvolvimento humano e buscando estratégias para intervir através da promoção e prevenção de saúde. Para os alunos dos cursos de saúde, em especial de Odontologia, é imprescindível uma formação mais ampla, em ambientes diferenciados, com experiências inovadoras, atividades coletivas e não somente a prática tradicional realizada em clínica de ensino.

\section{REFERÊNCIAS}

1. Scavuzzi AIF, Gouveia CVD, Carcereri DL, Veeck EB, Ranali J, Costa LJ et al. Revisão das 
diretrizes da ABENO para a definição do estágio supervisionado curricular nos cursos de Odontologia. Rev Abeno. 2015;15(3):109-13.

2. Albuquerque VS, Gomes AP, Rezende CHA, Sampaio MX, Dias OV, Lugarinho RM. A integração ensino-serviço no contexto dos processos de mudança na formação superior dos profissionais da saúde. Rev bras educ med. 2008;32(3):356-62.

3. Moyses ST, Moyses SJ, Kriger L, Schmitt EJ. Humanizando a educação em Odontologia. Rev Abeno. 2003;3(1):58-64.

4. Kriger L. Perfil profissional para o programa Saúde da Família. In: Carvalho ACP, Morita MC, Kriger L. Políticas Públicas para Educação e Saúde em Odontologia. São Paulo: Nupps/USP. 2007;11-8.

5. Ceccim RB, Feuerwerker LCM. O quadrilátero da formação para a área da saúde: ensino, gestão, atenção e controle social. Physis. 2004;14(1):4165.

6. Ceccim RB, Feuerwerker LCM. Mudança na graduação das profissões de saúde sob o eixo da integralidade. Cad. Saúde Pública. 2004; 20(5):1400-410.

7. Scharf D, Oliveira MF, Oliveira A, Schlindwein $\mathrm{CH}$, Rastelli MCS, Andrade ICGB. Odontologia itinerante na extensão universitária: FURBMóvel -promovendo saúde bucal e cidadania. Rev Bras Extensão Universitária. 2016;7(1):37-42

8. Nunes ALPF, Silva MBC. A extensão universitária no ensino superior e a sociedade. Mal Estar e Sociedade 2011;4(7):119-33.

9. Brustolin J, Brustolin J, Toassi RFC, Kuhnen M. Perfil do acadêmico de Odontologia da Universidade do Planalto Catarinense - Lages SC, Brasil. Rev Abeno. 2006;6:70-6.

10.Campostrini VL, de Carvalho RB, Daroz CBS, Daroz LGD, Sarcinelli A, Batitucci R. Formação profissional em odontologia: contribuição do programa atendimento à saúde bucal para a população de baixa renda - Vitória-ES. Rev Guará. 2015; (3):39-49.

11.Brasil. Resolução CNE/CNS 3/2002. Diretrizes Curriculares Nacionais do Curso de Odontologia. Diário Oficial da União 2002: S.1(42):10-11.

12. Oliveira JK, Vieira LE, Limeira MS, Araújo MG, Oliveira DH, Chacon LD at al. Práticas educativas em saúde bucal direcionada aos usuários do restaurante popular da cidade de Patos - PB. Rev Bras de Extensão Universitária 2017;8(2):67-74.

13.Ricci NA, Gonçalves DFF, Coimbra IB, Coimbra AMV. Fatores Associados ao Histórico de Quedas de Idosos Assistidos pelo Programa de Saúde da Família. Saúde e Sociedade. 2010;19(4):898-909.

14.Pereira SM, Mialhe L, Pereira LJ, Soares MF, Tagliaferro EPS, Meneghim MC et al. Extensão universitária e trabalho voluntário na formação do acadêmico em Odontologia. Arq Odontol. 2011;47(2):95-103.

15.Leme PAT, Pereira AC, Meneghim MC, Mialhe FL. Perspectivas de graduandos em odontologia acerca das experiências na atenção básica para sua formação em saúde. Ciênc saúde coletiva. 2015;20(4):1255-65.

16.Domingues GC, Fonseca GS, Zilbovicius C, Junqueira SR. Contribuições de estratégias extramurais para a formação em odontologia. Rev Bras Extensão Universitária 2016;7(2):105-11.

17.Loretto NRM, Silva IDPP, Batista LC. Clínica de atenção básica: do discurso teórico à realidade prática. Rev Abeno. 2010;10(2):20-26.

18.Pinheiro FMC, Nobrega Therrien SM, Almeida MEL, Almeida MI. A formação do cirurgiãodentista e a promoção de saúde no PSF. Rev Odontol UNESP. 2008;37(1):69-77.

\section{CONFLITO DE INTERESSES}

Os autores declaram não haver conflitos de interesse.

\section{AUTOR PARA CORRESPONDENCIA}

José Henrique de Araújo Cruz

henrique_araujo1992@ hotmail.com

Submetido em 07/10/2018

Aceito em 12/03/2019 